\title{
Community Genetic Services in Iran
}

\author{
Shirin Atri Barzanjeh, ${ }^{1}$ Mozhgan Behshid, ${ }^{1,2}$ Mohammad Bagher Hosseini, ${ }^{3}$ \\ Maryam Ezari, ${ }^{1}$ Mahdieh Taghizadeh, ${ }^{4}$ and Saeed Dastgiri ${ }^{4}$ \\ ${ }^{1}$ School of Nursing and Midwifery, Tabriz University of Medical Sciences, Tabriz 5166615739, Iran \\ ${ }^{2}$ Health System Research Committee, Tabriz Health Services Management Research Centre, Tabriz University of Medical Sciences, \\ Tabriz 5166615739, Iran \\ ${ }^{3}$ Pediatric Health Research Centre, Tabriz University of Medical Sciences, Tabriz 5166615739, Iran \\ ${ }^{4}$ Tabriz Health Services Management Research Centre, School of Medicine, Tabriz University of Medical Sciences, \\ Tabriz 5166615739, Iran
}

Correspondence should be addressed to Mozhgan Behshid, mozhganbehshid@hotmail.com

Received 27 July 2012; Revised 12 November 2012; Accepted 13 November 2012

Academic Editor: Maj Hulten

Copyright (C) 2012 Shirin Atri Barzanjeh et al. This is an open access article distributed under the Creative Commons Attribution License, which permits unrestricted use, distribution, and reproduction in any medium, provided the original work is properly cited.

The aim of the study was to report a description of the primary, secondary, and tertiary level services available for genetic disorders in Iran. For the purpose of this study, essential data were collected from every facility providing community genetic services in Tabriz city of Iran using a prestructured checklist. Technical information was filled in the predesigned forms using diagnostic records of each client/patient. Information was also gathered from community genetic services clients through a face-to-face interview at these facilities to assess the quality of services provided. Primary prevention measures were available in 80 percent of centres in the study population. Diagnostic techniques were fully available in the study area both in public and private sectors. Screening of congenital hypothyroidism and thalassemia has been successfully performed across the country by the Ministry of Health. Other screening programs have also been initiated by the country health authorities for neural tube defects, Down syndrome, and phenylketonuria. The high cost of genetic services at secondary and tertiary levels does not allow many people to get access to these services despite their needs. Governments will therefore need to allocate necessary resources to make the essential genetic services available for everyone needing these in the community.

\section{Introduction}

Prenatal genetic service has a key role in detecting the genetic risks and carrier screening for thehereditary conditions.

Prenatal genetic service has a key role in detecting the genetic risks and carrier screening for certain hereditary conditions such as sickle cell disease, cystic fibrosis, Tay Sachs disease, and alpha/beta thalassemia. According to the previous studies in the region, the genetic disorders and congenital abnormalities can affect individuals in different ages ranging from 2 percent at birth to 65 percent of individuals in their lifetime period. They account for approximately 30 percent of pediatric hospital admissions ranging from mild to very severe conditions $[1,2]$.

The essential goals of Community Genetic Services (CGS) are to manage the prevention, clinical, and psychosocial demands of individuals suffering from/at risk of hereditary disorders. This can be achieved through timely and precise clinical and laboratory diagnosis, genetic consultation, and primary health care services [3].

As for any disease, prevention methods in genetic disorders are performed at three levels including primary, secondary, and tertiary. Primary prevention is to avoid the occurrence of a disease (i.e., genetic consultation). Secondary prevention methods are to find and treat patients as early as possible (i.e., drug treatments for some heart anomalies). Tertiary prevention includes every effort to reduce the negative effects of the disease through treatment or rehabilitation (i.e., rehabilitation for limb anomalies).

The aim of the study was to report a brief description of the primary, secondary, and tertiary level services available for genetic disorders in Tabriz city of Iran as a sample pattern of CGS for similar regions. 


\section{Methods}

This study was carried out in Tabriz city of Iran. The city is one of the three major cities in the northwest of the country. Health houses, primary care centres, public/private clinics and hospitals, and Welfare and Rehabilitation Organization provide CGS in the area covering about 5 millions of population in the northwest of the Iran.

For the purpose of this study, essential data were collected from every facility providing CGS in the area using a prestructured checklist. Technical information was filled on the predesigned forms using diagnostic records of each client/ patient. Information was also gathered from CGS clients through a face-to-face interview at these facilities to assess the quality of services provided.

The study took place between March and June 2010. A total number of 19 public and private hospitals and clinics and 15 heath centres were checked for the purpose of this study. A total number of 140 clients/patients were interviewed to assess the quality of services, costs, and so forth.

Approval for this study was obtained from the Regional Committee of Medical Ethics of Tabriz University of Medical Sciences. Accordingly, an informed consent from the study participants was obtained and data were gathered anonymised at the source of data collection to keep personal information private.

\section{Results}

Some of the current services at primary, secondary, and tertiary levels available for genetic disorders in Iran are listed in Table 1.

Primary prevention measures were available in 80 percent of centres and facilities in the study population. These measures include preconceptional care and folic acid supplementation, pregnancy health care services, maternal and children health care services, vaccination (i.e., rubella, measles, etc.), and various educational programmes for the primary prevention of congenital anomalies and genetic disorders.

Diagnostic techniques including ultrasonography, DNA extract tests (including PCR), serum alpha fetoprotein, nonconjugated estradiol, amniocentesis, chromosomal analysis of amniotic fluid, maternal phenyl alanine test, urine and plasma amino acids analysis fetal echocardiography, MRI scan and CT scan, electroencephalography, chest radiography, electrocardiogram, different type of echocardiography, color ultrasonography, pulse and color Doppler, angiocardiography, pulmonary angiography, liver function tests, IV urography and urethrography, blood gas analysis, chest radiography, radiography by barium swallow, thoracentesis, bronchography, lung function tests, lung biopsy, lung aspiration, bronchoscopy, laryngoscopy, thoracoscopy, radionucleotide scan, fluoroscopy, arteriography, and aortography were all fully available in the study area both in public and private sectors.
Screening of congenital hypothyroidism and thalassemia has been successfully performed across the country by the Ministry of Health [4]. Other screening programs have also been planned/started by the country health authorities for neural tube defects, the Down syndrome, and phenylketonuria.

Selected types of congenital anomalies (i.e., heart defects) were routinely treated by various types of drugs as well as the neurosurgical technique for hydrocephaly and Spina Bifida, shunting for hydrocephaly with or without myelomeningocele, reconstructive surgery for cleft lip, and palate and for patent ductus arteriosus (PDA), ventricular septal defect (VSD), Atrioventricular (AV) canal, tetralogy of fallot, pulmonary valve stenosis and noncyanotic congenital heart diseases, urology corrective surgery for hypospadias, cryptorchidism, undiscerning testis, arterioplasty for coarctation aorta, valvuoplasty and valve replacement for aortic valve stenosis, craniosynostosis surgery, dialysis and kidney transplants for congenital renal diseases, and bone marrow transplantation for different type of bone marrow failure.

\section{Discussion}

In this study, we reported the current status of genetic services for the prevention, treatment, and rehabilitation of congenital anomalies in Iran provided by public and private sectors in the area.

This study showed that primary prevention of congenital anomalies and genetic disorders (i.e., folic acid supplementation before and during pregnancy, etc.) is now well established in the region. Despite some differences, similar findings have been reported in previous studies [5-7] indicating that the activities by the Ministry of Health and private sectors for the prevention of genetic disorders are well understood/accepted in the country. Research reports from the nine European countries, Georgia, Venezuela, and India showed that primary prevention measures have been implemented in these regions too [8-11]. However, the structure of the preventive strategies may vary by the region based on the population demands and financial sources.

Our study indicated that despite availability of diagnostic and treatment facilities for genetic disorders in the area, they are mostly too expensive to be fully accessible for many people in the country as they responded in the face-to-face interview. It is obvious that as more advances in genetic technology occurring, the demands for CGS are growing in the population. However, the high cost of genetic services does not allow many people to get access to these services despite their needs. Governments will therefore need to allocate necessary resources to make the essential genetic services available for everyone needing that in the community. More investigations are needed to assess the cost effectiveness and health economics aspects of this matter.

\section{Conflict of Interests}

The authors declare that they have no competing interests. 
Table 1: Community Genetic Services in Iran.

\begin{tabular}{|c|c|}
\hline Services & Congenital abnormalities \\
\hline Alpha fetoprotein & Neural tube defects, nephrosis, chromosomal abnormalities, omphalocele \\
\hline Amniocentesis & Neural tube defects, chromosomal abnormalities \\
\hline Amniotic fluid chromosomal analysis & Trisomy 13, 18, 21 and chromosomal abnormalities \\
\hline Aortography & Congenital heart abnormalities \\
\hline Arteriography & Congenital heart abnormalities \\
\hline Arterioplasty with balloon & Coarctation aorta \\
\hline Blood gas analysis & Congenital respiratory diseases \\
\hline Bone marrow transplantation & Hydroxyurea \\
\hline Bronchography & Congenital respiratory diseases \\
\hline Bronchoscopy & Congenital respiratory diseases \\
\hline Chest radiography & Congenital respiratory diseases \\
\hline Chest radiography & Congenital heart abnormalities \\
\hline Chorionic gonadotropin level & Trisomy $13,18,21$ \\
\hline Color ultrasonography and ventriculography & Congenital heart abnormalities \\
\hline Cordocentesis services & Hemoglobinopathies, fetal anemia, acid-base disorders, thalassemia \\
\hline Craniosynostosis surgery & Nervous system anomalies \\
\hline Dialysis and kidney transplants & Congenital renal diseases \\
\hline DNA services & $\begin{array}{l}\text { Thalassemia, muscular dystrophy, phenylketonuria (PKU), Turner syndrome, } \\
\text { cystic fibrosis }\end{array}$ \\
\hline Drug treatments & Congenital heart abnormalities \\
\hline Echoencephalography procedure & Neural tube defects \\
\hline Electrocardiogram & Congenital heart abnormalities \\
\hline Embryo echocardiography & Congenital heart abnormalities \\
\hline Embryoscopy & Facial and limb abnormalities \\
\hline Exercise testing, congenital heart disease & Congenital heart abnormalities \\
\hline Fetal skin biopsy & Albinism \\
\hline Fetoscopy & Limb abnormalities \\
\hline Fluoroscopy & Congenital heart abnormalities \\
\hline Genital ambiguous surgery & Urogenital malformations \\
\hline Growth monitoring & Congenital anomalies diagnosed after birth \\
\hline Hemoglobin electrophoresis & Sickle cell anemia \\
\hline Herpes and cytomegalovirus tests & Mother and fetus \\
\hline Intrauterine surgery & Diaphragmatic hernia and myelomeningocele \\
\hline Iron levels and hemoglobin electrophoresis & Thalassemia \\
\hline IV urography and urethrography & Obstructive urethral congenital disease \\
\hline Laryngoscopy & Congenital respiratory diseases \\
\hline Liver function tests & Hemochromatosis \\
\hline Lung aspiration & Congenital respiratory diseases \\
\hline Lung biopsy & Congenital respiratory diseases \\
\hline Lung function tests & Congenital respiratory diseases \\
\hline Maternal health care services & Congenital anomalies \\
\hline Maternal phenyl alanine & Fetal microcephaly \\
\hline MRI scan and CT scan & Neural tube defects (hydrocephaly and spina bifida) \\
\hline Neurosurgical techniques & Hydrocephaly and Spina Bifida \\
\hline
\end{tabular}


TABle 1: Continued.

\begin{tabular}{|c|c|}
\hline Services & Congenital abnormalities \\
\hline NICU and special delivery services & Pregnancies with omphalocele \\
\hline Nonconjugated acetyl test & Trisomy 21 \\
\hline Preconceptional folic acid consumption & Congenital anomalies \\
\hline Pregnancy health care services & Congenital anomalies \\
\hline Public education & Congenital anomalies \\
\hline Pulse and color doppler, angiocardiography & Congenital heart abnormalities \\
\hline Radiography by barium swallow & Congenital respiratory diseases \\
\hline Radio-nucleotide scan & Congenital respiratory diseases \\
\hline Reconstructive surgery & $\begin{array}{l}\text { VSD, PDA, AV, tetralogy of fallot, pulmonary valve stenosis, and noncyanotic } \\
\text { congenital heart diseases }\end{array}$ \\
\hline Reconstructive surgery & Cleft lip and palate \\
\hline Screening programme & Congenital hypothyroidism, adrenal hyperplasia \\
\hline Shunting & Myelomeningocele with hydrocephaly \\
\hline Surgical treatment & Neurofibromatosis \\
\hline Thoracentesis & Congenital respiratory diseases \\
\hline Thoracoscopy & Congenital respiratory diseases \\
\hline Treatment by dexamethasone & High risk pregnancy for congenital adrenal hyperplasia \\
\hline Two-dimensional echocardiography & Congenital heart abnormalities \\
\hline Ultrasound diagnostic services & Neural tube defects, heart defects, diaphragmatic hernia \\
\hline Urine and plasma amino acids, serum ammonium & Fetal microcephaly \\
\hline Urology corrective surgery & Hypospadias, cryptorchidism, undescending testis \\
\hline Vaccination & Congenital defects caused by viral infections \\
\hline Valvuloplasty and valve replacement & Aortic valve stenosis \\
\hline Varios clinical tests & Eye defects, thyroid anomalies, speech and hearing defects \\
\hline
\end{tabular}

\section{Acknowledgments}

The authors are grateful to the Research Deputy of the Tabriz University of Medical Sciences and National Public Health Management Centre and Pediatric Health Research Centre for supporting this study. The authors are also grateful to all clients/patients for sharing their experiences with us, and to health houses, primary care centres, public/private clinics and hospitals, and Welfare and Rehabilitation Organization for participating in this study.

\section{References}

[1] L. Al-Gazali, H. Hamamy, and S. Al-Arrayad, "Genetic disorders in the Arab world," British Medical Journal, vol. 333, no. 7573, pp. 831-834, 2006.

[2] G. Tadmouri, Genetic Disorders in Arab Populations, UAE: Centre for Arab Genomic Studies Publications, 2008.

[3] R. Harris, "Concerted action on genetic services in Europe: a comparative study of 31 countries," European Journal Human Genetics, vol. 5, pp. 1-220, 1997.

[4] H. Aghajani, A. Samavat, M. Haghazali, F. Valizadeh, and G. Sarbazi, "Primary health care: an approach to community control of genetic and congenital disorders," Iranian Journal of Public Health, vol. 38, no. 1, pp. 113-114, 2009.

[5] M. Mirmohammadaliee, M. Modares, G. Babai et al., "The effect of education on awareness and use of folic acid supplementation in pregnant women," Haiat Journal, pp. 38-31, 2004.
[6] Z. Safdari and F. Ghodsi, "Effect of education on knowledge of the role and use of folic acid supplementation in pregnant women," Ghazvin Journal of Medical Sciences, vol. 12, pp. 3-4, 2008.

[7] M. Shamsi and A. Baiaty, "The effect of education on knowledge, attitude and practice of pregnant women referred to health centers in Arak," Gonabad Journal of Medical Sciences, vol. 15, pp. 27-34, 2009.

[8] B. Langer, M. P. Caneva, and G. Schlaeder, "Routine prenatal care in Europe: the comparative experience of nine departments of gynaecology and obstetrics in eight different countries," European Journal of Obstetrics Gynecology and Reproductive Biology, vol. 85, no. 2, pp. 191-198, 1999.

[9] P. J. Meehan, "Congenital anomalies in georgia," Georgia Epidemiology Report, 1996.

[10] S. González-Ferrer, L. Pineda-Bernal, W. Delgado-Luengo, and $\mathrm{H}$. Villalobos-Cabrera, "Medical genetics in Zulia, a state of Venezuela," Community Genetics, vol. 7, no. 2-3, pp. 153156, 2004.

[11] I. C. Verma, R. Saxena, M. Lall, S. Bijarnia, and R. Sharma, "Genetic counseling and prenatal diagnosis in India-experience at Sir Ganga Ram Hospital," Indian Journal of Pediatrics, vol. 70, no. 4, pp. 293-297, 2003. 

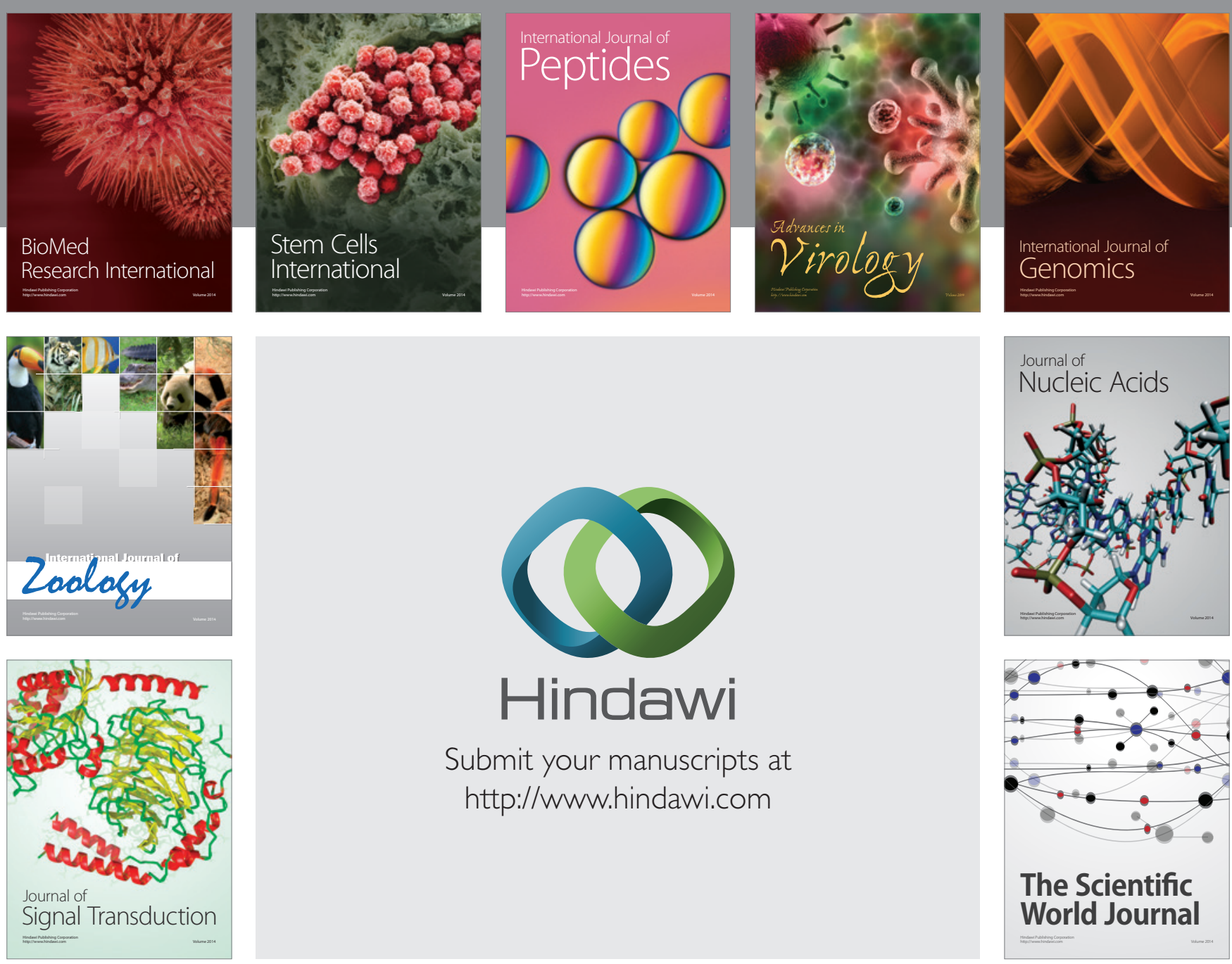

Submit your manuscripts at

http://www.hindawi.com
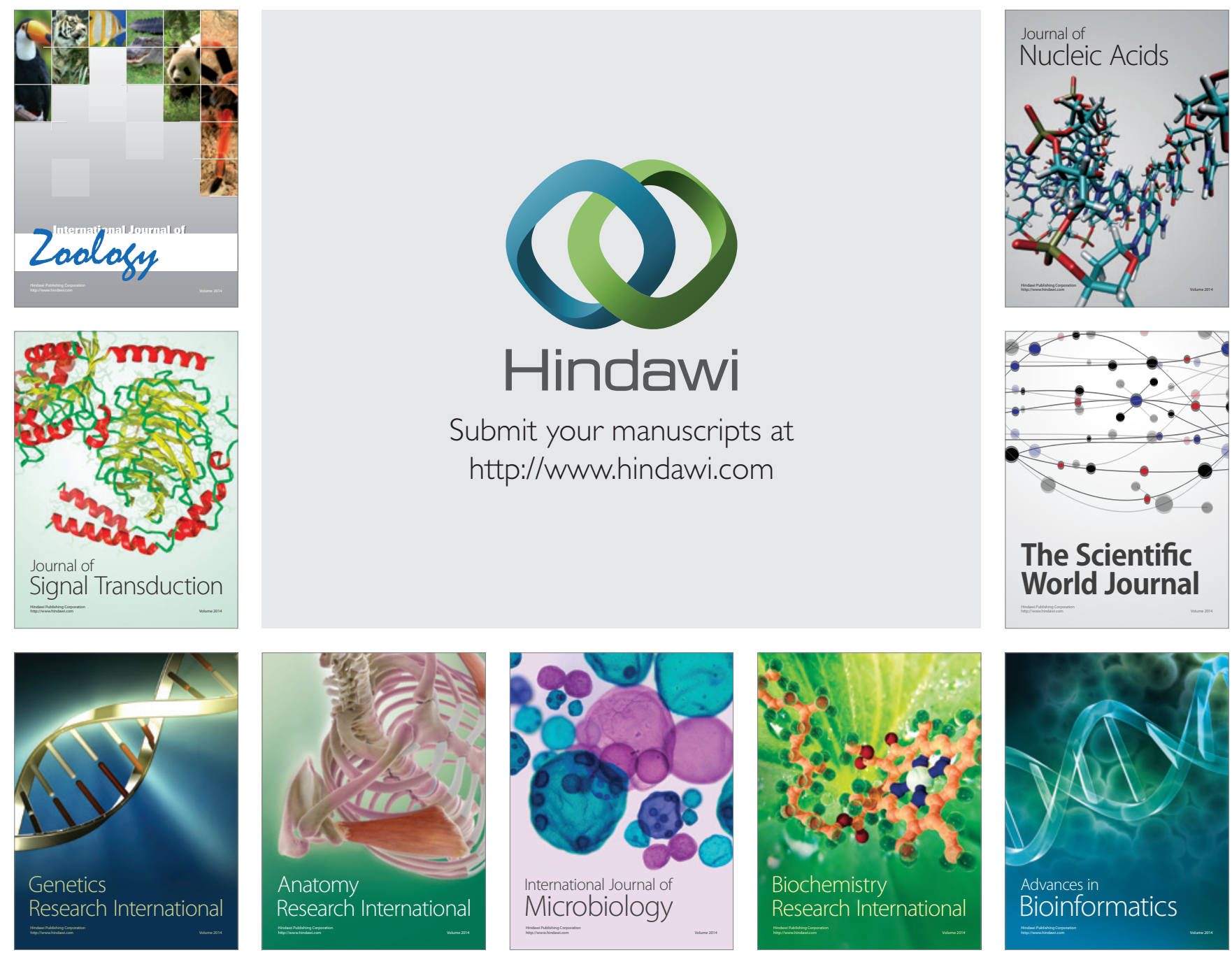

The Scientific World Journal
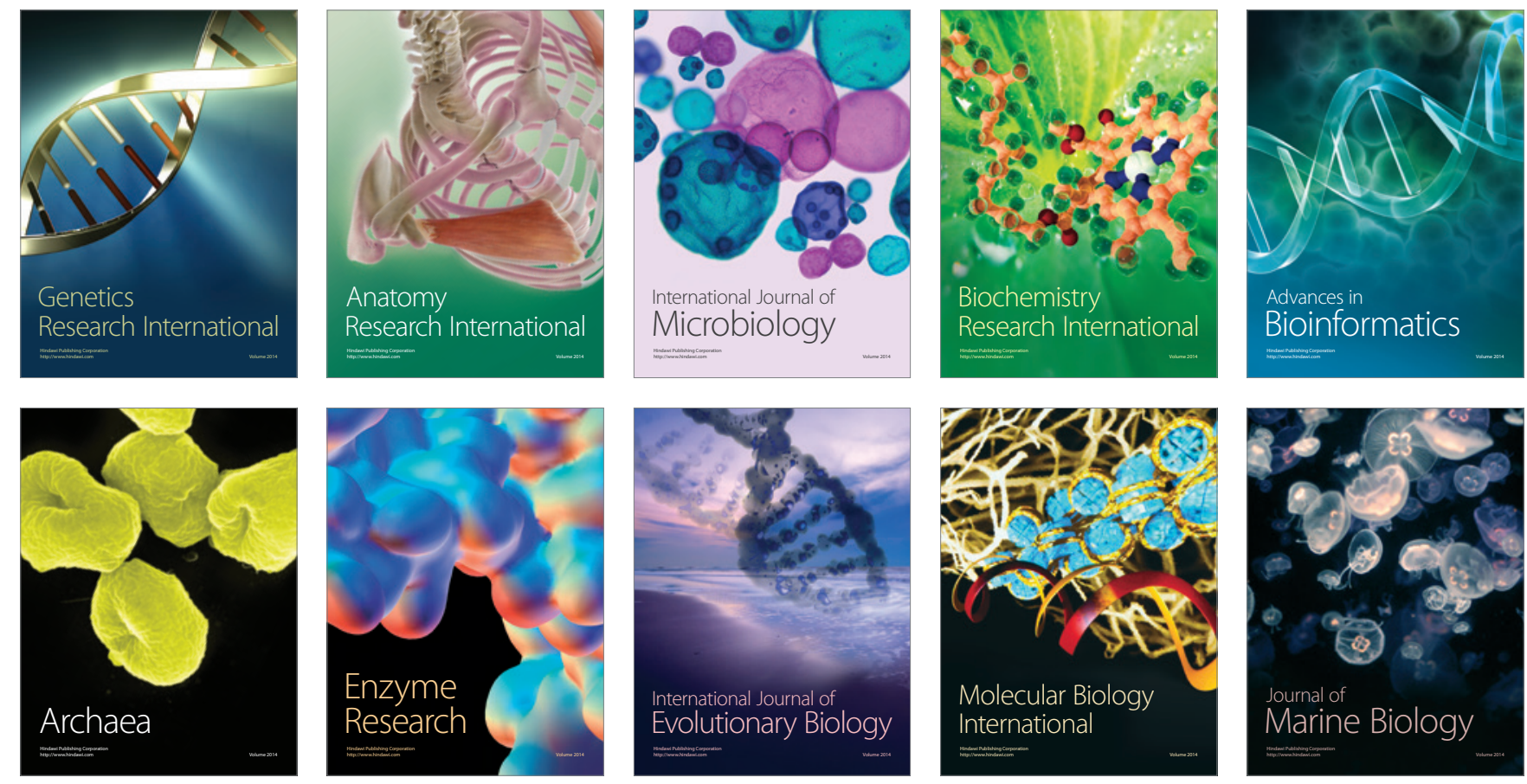